\title{
Antisocial and Schizoid Personality Disorder Scales: Conceptual bases and preliminary findings
}

\author{
Octav - Sorin Căndel ${ }^{1} \&$ Ticu Constantin ${ }^{1}$ \\ ${ }^{I}$ Department of Psychology, "Alexandru Ioan Cuza” University of Iaşi, Romania
}

Received 12.09.2016; Received revised 03.06.2017; Accepted 19.06.2017

Available online 30.06 .2017

\begin{abstract}
The study describes the development and validation of two scales which can be used in evaluating schizoid and antisocial personality disorders. Both scales were developed relying on descriptions from DSM 5 and ICD 10. For validation, the scales have been tested on 125 subjects, together with two well-known psychometric instruments, DA ${ }^{12 p r o f i l e}$ Personality Inventory, and SCL-90. Internal consistency is calculated using Cronbach's alpha coefficient. Schizoid Scale contains 20 items and shows a good internal consistency (Cronbach's $\alpha=.77$ ) and Antisocial Scale contains 22 items and has excellent internal consistency (Cronbach's $\alpha=.91$ ). The correlations between the scores of the two scales and the scores of DA ${ }^{12 p r o f i l e}$ Personality Inventory and SCL-90 are statistically significant. The factorial analysis reveals that the two scales and DA ${ }^{12 p r o f i l e}$ Personality Inventory sub-scales are clustered in four factors, explaining $68.31 \%$ of the variance. Based on these results, we discussed the importance the scales have for psychological research and for psycho-diagnostic, their limitations and our future directions of research.
\end{abstract}

Keywords: Antisocial, Schizoid, Personality Disorders, Scale.

Address of correspondence: Ticu Constantin, Department of Psychology, Alexandru Ioan Cuza University of Iasi, Toma Cozma street, no.3, Iasi, 700554, Romania.

E-mail: tconst@uaic.ro

Acknowledgement: We would like to acknowledge all the students from the "PsyPro" research group. Their creativity and understanding were invaluable to the development of the scales.

\section{Introduction}

The problem of personality disorder is largely discussed in psychiatry and psychology. Nevertheless, we cannot properly understand disorder or abnormality without a view of the normal development of personality; in this context, the current trend is to integrate the classification of personality disorders in a dimensional model of general personality structure, such as The FiveFactor Model (FFM; Samuel \& Widiger, 2008; Widiger \& Trull, 2007).

From this perspective, to develop new instruments for personality evaluation (questionnaires, check-lists, structured interviews etc.) is essential to identify the most appropriate theoretical model where the two aspects (normality and abnormality) are properly represented.

Normal personality and personality disorders

In recent years, two different frameworks have tried to explain the normal personality and personality disorders. The categorical model is the medical model, followed by psychiatrists and psychologists as well. It assumes that normality and abnormality are two qualitatively different states. The Diagnostic and Statistical Manual of Mental Disorders (5th ed.; DSM-5; American Psychiatric Association, [APA] 2013) defines personality disorder as "an enduring pattern of inner experience and behavior that deviates markedly from the expectations of the individual's culture, is pervasive and inflexible, has an onset in adolescence or early adulthood, is stable over time, and leads to distress or impairment" (p. 645). This pattern manifests in the following areas: cognition (i.e., ways of perceiving and interpreting self, other people, and events), affectivity (i.e., the range, intensity, lability, and appropriateness of emotional response), interpersonal functioning or impulse control (APA, 2013).

The other framework for personality disorders - the trait perspective - is based on different scientific discoveries that support the idea that personality structure is identical regardless of clinical or non-clinical samples, both normal and abnormal states are related at the etiological level and that personality disorders are some 
extreme variants of normal personality (Markon, Krueger \& Watson, 2005). The most quoted model of this framework is the Five Factor Model (Widiger \& Costa, 1994; Widiger \& Trull, 2007; Samuel \& Widiger, 2008).

Personality assessment remains one of the most important responsibilities of both psychiatrists and psychologists. The normal personally measurements are requested by employers, schools or in the medical system, while the assessment of the personality disorders is conducted to differentiate normal and well-adapted persons from the people at risk, who are in need of professional treatment.

Specialists use two main methods to assess personality. In the fields of organizational, industrial, counseling or educational psychology (and in a lesser extent in the clinical field), professionals use personality tests more frequently. Conversely, clinical psychologists and psychiatrists use clinical interviews and observation.

To respond to the need for evaluating different aspects of personality in between normality and pathology, we developed two instruments in order to measure the symptomatology of Antisocial Personality Disorder and Schizoid Personality Disorder. We did it in agreement with DSM V descriptions (5th ed.; DSM-5; American Psychiatric Association, 2013) and in order to integrate these scales in an already validated assessment tool: $D A^{12 p r o f i l e}$ Personality Inventory (Constantin, Hojbotă, Niculescu, Nechita, Amariei \& Macarie, 2010).

\section{Antisocial Personality Disorder}

Antisocial Personality Disorder (ASPD in DSM-5) is included in Cluster B of personality disorders, along with the Borderline, Histrionic, and Narcissistic PDs. The cluster characterizes individuals with an erratic, emotional or overly dramatic behavior (APA, 2013).

The first description of ASPD in the DSM appeared in its third edition (APA, 1980). By that time, the specialists had used a large variety of labels to describe individuals that are prone to a pattern of disregard for and violation of the rights of others, the most widely used being "Psychopathy" and "Sociopathy". Kraepelin called them "psychopathic personalities", while Clenkley described the psychopaths as "individuals who combined deviant personality traits and antisocial behaviors which were often criminal in their severity" (Sutker \& Allain, 2002, p. 446). However, the term psychopathy did not appear in any DSM unto its third edition. The closest disorder to the current ASPD was sociopathy or "sociopathic personality", a disorder that found its place in the first edition of the DSM (APA, 1952) and used to characterize the individuals who did not profit from experience or punishment and maintained no real loyalties (Pickersgill, 2012, as cited in Houser, 2015). Since the first real description of ASPD (1980), the criteria for the diagnosis have remained mostly the same (Sutker \& Allain, 2002).

In the current edition of DSM (V), Antisocial Personality Disorder is described as "a pervasive pattern of disregard for and violation of the rights of others, occurring since age 15 years" (APA, 2013, p. 659). The manual offers a series of criteria for the diagnosis, such as irresponsibility, irritability, a history of lying or conning, aggressiveness, lack of remorse, trouble with the law or reckless behavior that puts his life or others' lives in danger. The person is unable to find or maintain a job and is prone to lawless acts such as violence, theft, fraud etc. The individuals diagnosed with such disorder are of 18 years or older and have been characterized by symptoms of conduct disorder. Conduct disorder involves a "repetitive and persistent pattern of behavior in which the basic rights of others or major age-appropriate societal norms or rules are violated. The specific behaviors characteristic of conduct disorder falls into one of four categories: aggression to people and animals, destruction of property, deceitfulness or theft, or serious violation of rules" (APA, 2013, p.659).

In the World Health Organization's ICD-10, the ASPD is called dissocial personality disorder. It is described as a disorder characterized by the disregard for social obligations, and unconcern for the feelings of others. Individuals behave in an aggressive way and are not affected by any form of punishment or guilt. They usually break social norms and have the tendency of blaming others for their own problems (ICD-10, 1992). Despite the difference in terminology, the two PDs are mostly the same.

After publishing the DSM criteria, researchers and clinicians needed a valid and reliable way to differentiate between clinical and non-clinical groups. In order to assess such differences, they use a multitude of clinical structured interviews, behavior and symptom checklists and selfreported questionnaires. Hare $(1980,1985)$ created some of the most important ways of assessment, such as The Psychopathy Checklist or the Self-Report Psychopathy Scale. Another well known inventory is the MCMI-III, a multiscale inventory aiming at evaluating personality disorders (Milton, 1994, as cited in Sutker \& Allain, 2002). Still, the scales for the assessment of ASPD are rather scarce and the existent ones are either very long or must be used with a behavioral checklist (such as the SRP).

\section{Schizoid Personality Disorder}

Schizoid Personality Disorder (SPD), along with the Paranoid and Schizotypal Personality Disorders are part of the Cluster A. Individuals diagnosed with one or more of these disorders are known for their eccentric behavior and their lack of social relationships. Another resemblance is that all three disorders correlate with a high rate of schizophrenia in the family of origin (Miller, Useda, Trull, Burr \& Minks-Brown, 2002).

Historically, SPD was described as a lower form of schizophrenia, named pseudoneurotic schizophrenia by Hoch (1910) or latent schizophrenia by Bleuler (1922). Up until the 1970s, the term Schizoid was used to describe a mental state close to schizophrenia (schizophrenia-like), but later the change in terminology transferred this meaning to Schizotypal PD. Ribot, in 1890, used the term "anhedonia" and so he gave the first description of SPD, talking about individuals that find no physical or social pleasures. SPD also appeared in the first edition of DSM (APA, 1952), but its description was very short, with only three characteristic traits: avoidance of close relationships, inability to express any kind of aggressiveness and autistic thinking. Only in DSM-3 (APA, 1980), SPD received a broader description and the first diagnostic criteria. The third edition of the manual also brought another PD, Avoidant, similar to SPD. Both PDs are characterized by a lack of social relationships, but the motives behind this are different. APD diagnosed individuals are socially anxious, while the ones who suffer from SPD are indifferent and aloof (Miller et al., 2002).

Nowadays, the fifth edition of DSM described SPD as a pattern of social detachment characterized by a low range of emotions in interpersonal context. For a diagnostic, an individual must have four of the following characteristics: 
a lack of interest in social, romantic, family relationships, no interest in sexual activity, a predisposition to very few and solitary activities, indifference to criticism or praise, emotional coldness and detachment. Like all PD, SPD is diagnosed after the age of 18 (APA, 2013).

ICD-10 (1992) also insists on the lack of interest in any social or emotional contact. Also, they add a predisposition to fantasy and introspection and say that individuals with SPD might have difficulties in expressing feelings and in experiencing pleasure.

One of the main problems with the categorical approach of personality disorders is the lack of clarity about the assessments. In the DSM, the main difference between normality and the presence of the disorder is given by expressions such as "little if any" or "almost always". In order to overcome such criticism, specialists have created alternative means of evaluation (Miller et al., 2002). One of the most important is the use of FFM and its specific tests, such as the NEO-PIR (Costa \& McCrae, 1992). Widiger, Trull, Clarkin, Sanderson and Costa (1994) predicted that SPD would associate with low scores on Warmth, Gregariousness, Excitement Seeking, and Positive Emotions (dimensions of Extraversion), Hostility and Self-Consciousness (Neuroticism), Feelings (from Openness).

\section{The current study}

We tried to address one of the most important issues in the Romanian psychiatric system, the lack of reliable instruments validated for the Romanian population in regards to the assessment of Personality Disorders as described by the Diagnostic and statistical manual of mental disorders (APA, 2013) and the International Statistical Classification of Diseases and Related Health Problems (ICD-10, 1992). Therefore, our main goal was to develop two reliable instruments for the evaluation of the two personality disorders above mentioned: Antisocial Personality Disorders and Schizoid Personality Disorder, modeled on the DSM-V (APA, 2013) and ICD 10 (1992) criteria for the PDs. We also wanted to integrate the present scales into an existing personality questionnaire, the DA ${ }^{12 p r o f i l e}$ Personality Inventory (Constantin et al., 2010).

Our second goal was to verify the psychometric properties of the proposed tools, such as reliability and construct validity. We focused on verifying the relationship between our two scales and other self-reported measures of personality, The DA ${ }^{12 \text { profile }}$ Personality Inventory (Constantin et al., 2010) and Symptom Checklist-90 (Derogatis, 1977).

DA $12^{\text {profile }}$ Personality Inventory is a useful tool for this analysis for two reasons. Primarily, it was built from the perspective of a competing model to the one in which DSM and ICD derived: Kleist-Leonhard classification system (Nosology). Secondly, because it is focused on the evaluation of similar traits ("accentuated traits"; Leonhard, 1970) described as existing between functional normality and malfunctioning pathological (Leonhard, 1970).

Also, we wanted to study the correlation between the two scales that were constructed using DSM and description ICD - ASPD and SPD -, and a scale that evaluate general symptomatology. SCL-90 was chosen because it is one of the main evaluation methods used in Romanian psychiatric system and it proved to be a reliable instrument assessing psychiatric symptoms (Holi, 2003).

\section{Method}

\section{Item generation}

Our goal was to develop two instruments that can assess all the diagnostic criteria for the two PDs based on both the DSM-V and ICD-10 manuals. In order to achieve such a goal, we assembled an expert group of students, practicing psychologists, psychiatrists and academics. The main sources for generating items were the traits description from DMV-V and ICD-10. Each member proposed a number of items based on the descriptions given by the manuals. Our requirement was that all items comply with the same pattern: to be short, simple, specific, positive (not reversed) and to assess only one trait or behavior. The majority of them were formulated as first person statements that reflect behaviors, cognitions or emotions, and others describe opinions on different topics. We tried to avoid general truths or references to other person beliefs. Each item has a dichotomous response, "yes" or "no". Initially, each scale consisted of more than 30 items, so we did a supplementary analysis, and eliminated the items that had the same meaning. In the end, both scales were reduced to 22 items each. The ASPD scale contains items such as "Only weak people need the protection of the law" or "When I do something, I do not take into consideration other people's opinions or emotions". Among the items of the SPD scale one can find, for example: "I think that emotions and feelings are overrated", "I do not feel at ease when I have to express my emotions or feelings".

\section{Participants}

Both scales in their final version were filled out by 125 participants, aged18 to $92(\mathrm{M}=37.35, \mathrm{SD}=15.18)$. They were recruited from the general population and from several Romanian counties (Iaşi, Neamţ, Bacău, Galaţi, Suceava, Vaslui). Regarding gender, 64 (51.2 \%) were females and $61(48.8 \%)$ were male. Regarding education, $32(25.6 \%)$ have completed 8th grade, $52(41.6 \%)$ have graduated high school, 39 (31.2\%) had a bachelor's degree and $2(1.6 \%)$ had a master's degree or higher. The scales were distributed by the authors and were completed at home by the participants. Also, all questionnaires were anonymous and confidential.

Due to the fact that symptoms of personality disorders lay between functional normality and dysfunctional pathology (Samuel \& Widiger, 2008), a first step in validating the ASPD and SPD scales was checking the psychometric characteristics in the normal population. Previous studies had shown that PDs have a high prevalence in the general population. Although we do not have data for Romania, researchers reported a prevalence of $11.1 \%$ (Ekselius, Tillfors, Furmark, \& Fredrikson, 2001) in Sweden, $14.79 \%$ (Grant et al., 2004) the United States and $13.4 \%$ (Torgersen, Kringlen, \& Cramer, 2001) in Norway.

\section{Measures}

The ASPD scale is a self-report scale that measures the symptoms of Antisocial Personality Disorder. It was modeled after the DSM V (2013) and ICD-10 (1992) criteria for the disorder. It contains 22 items with a dichotomous response (Yes/No). The SPD scale is also based on the traits description from DMV V and ICD-10. It measures the level of schizoid symptomatology using 22 dichotomous items (Yes/No). The psychometric properties of the two scales are highlighted in the present study. 
$D^{12 p r o f i l e ~ P e r s o n a l i t y ~ I n v e n t o r y ~(C o n s t a n t i n ~ e t ~ a l ., ~}$ 2010) is a self-report scale based on Karl Leonhard theory (1979) about accentuated personalities. It contains 151 items with dichotomous responses (True/False) and allows us to assess 13 accentuated dimensions of personality: demonstrativeness, hyper exactness, hyper-perseverance, uncontrollability, hyperthymia, dysthymia, lability, exaltation, anxiety, emotivity, dependency, neuroticism, and desirability. In was developed between 2005 and 2008 and was validated on Romanian Population. The DA ${ }^{12 p r o f i l e}$ Personality Inventory was compared with the similar, German inventory, developed by Schmieschek (1970); it has been shown to be superior in terms of internal consistency and test-retest reliability. In this study, Cronbach's Alfa coefficient ranged from .62 (for Dysthymia) to .82 (for Uncontrollability).

Symptom Check List-90 (Derogatis, 1977) is a selfreport questionnaire used for the assessment of various psychiatric problems and symptoms of psychopathology. It is based on Hopkins Symptom Checklist (HSCL) and includes 90 items, clustered into 9 specific factors (somatization, obsessive-compulsive, interpersonal sensitivity, depression, anxiety, hostility, phobic anxiety, paranoid ideation, psychoticism) and 3 general factors of psychological impairment. Psychological problems encountered in the last week (seven days) are evaluated on a Likert-type scale, with answers ranging from 0 ("not at all") to 4 ("extreme"). In the previous studies, SCL-90's internal validity have been generally good (ranging from .77 to .90$)$ and the authors have reported a decent validity (Holi, 2003). In the current study we used only the nine specific factors and found a good internal consistency for each of them. Cronbach's Alfa coefficient ranged from .76 (for Hostility) to .88 (for Somatization).

\section{Results}

\section{Reliability analysis}

In order to test the reliability of the two scales, we used Cronbach's Alfa coefficient. Both scales have demonstrated a good internal consistency, as reported in Table 1. For the Schizoid Scale $(\mathrm{M}=.39$, $\mathrm{SD}=$ .18 ), Cronbach's Alfa $=.743$, while for the Antisocial Scale $(\mathrm{M}=.25, \mathrm{SD}=.02)$, Cronbach's Alfa $=.845$. However, we considered that the coefficient for the Schizoid Scale could have been improved, so we decided to analyze each item and to remove the ones responsible for its limitation. Two items were removed and, as a result, the new analyses revealed a satisfactory growth of the coefficient. Nevertheless, the Schizoid Scale Cronbach's Alfa could not exceed the threshold of .800 .

\begin{tabular}{llll}
\multicolumn{5}{l}{ Table 1. Reliability of ASPD and SPD } \\
$\begin{array}{llll}\text { ASPD Scale } \\
(22 \text { items })\end{array}$ & $\begin{array}{c}\text { SPD Scale } \\
(22 \text { items })\end{array}$ & $\begin{array}{c}\text { SPD Scale } \\
(20 \text { items })\end{array}$ \\
\hline $\begin{array}{l}\text { Cronbach's } \\
\alpha\end{array}$ & .845 & .743 & .772 \\
\hline
\end{tabular}

\section{Correlations analysis}

We made two sets of correlations between SPD and ASPD scales, and each dimension of the $\mathrm{DA}^{12 \text { profile }}$ and SCL-90. In order to control for the social desirability bias, we used partial correlations instead of zero-order correlation. The controlled variable was the Desirability factor of DA ${ }^{12 \text { profile }}$. Table 2 displays the partial correlation coefficient between the PDs scales and the Accented
Dimensions results. ASPD and SPD are correlated with each other at a medium level $(\mathrm{r}=.637)$. Based in this result, we tested our data to determine if a common method bias may cause the correlation pattern. For this, we used Herman Single Factor Analysis (Herman, 1960). This technique can be conducted using an exploratory factor analysis. We loaded all items accounting for SPS and ASPD into a single factor unconstrained by any rotation. The variation explained by this factor was $17.027 \%$. Commonly, the literature says that the factor must explain more than $50 \%$ of the variance in order to assume a common method bias. Based on above results, we could conclude that the correlation pattern between the two scales is not generated by a common method bias. In the end, we computed a Global Severity Index, which is the mean value of all the items of SCL-90 (Holi, 2003). We conducted a partial correlation between the SPD score and the ASPD score with the above mentioned Index as a control variable. The correlation remained significant and the effect decreased, but only marginal $(r=.590, \mathrm{p}<.001)$.

Table 2. Partial Correlations between PDs scales and $\underline{\mathrm{DA}^{12 \text { profile }}}$ dimensions with DA-Desirability as control variable

\begin{tabular}{lll}
\hline & SPD & ASPD \\
\hline SPD & 1 & $0,635^{* *}$ \\
ASPD & $0,635^{* *}$ & 1 \\
DA-Demonstrativeness & 0,167 & $0,367^{* *}$ \\
DA-Hyper exactness & $0,181^{*}$ & 0,158 \\
DA-Hyper-perseverance & $0,416^{* *}$ & $0,595^{* *}$ \\
DA-Uncontrollability & $0,313^{* *}$ & $0,444^{* *}$ \\
DA-Hyperthymia & $0,237^{*}$ & $0,214^{*}$ \\
DA-Dysthymia & $0,398^{* *}$ & $0,206^{*}$ \\
DA-Lability & $0,306^{* *}$ & $0,36^{* *}$ \\
DA-Exaltation & 0,151 & $0,257^{*}$ \\
DA-Anxiety & $0,345^{* *}$ & $0,242^{*}$ \\
DA-Emotivity & 0,042 & 0,128 \\
DA-Dependency & $0,214^{*}$ & $0,378^{* *}$ \\
DA-Neuroticism & $0,392^{* *}$ & $0,28^{* *}$
\end{tabular}

Notes: $* p<.05 ; * * p<.01$.

As expected, SPD correlated with Hyper-perseverance, Dysthymia, Anxiety, and Neuroticism. Although significant, all the coefficients were rather weak, none of them exceeding .50. Also, very low correlations were found between SPD and Lability, Hyperthimia and Uncontrolability. In a similar analyses, medium expected correlation was found between ASPD and Hyperperseverance Uncontrollability, Lability and Dependency and low correlation with Hyperthimia, Dysthymia, Exaltation and Anxiety,

In order to verify the relationship between the ASPD, SPD and SCL-90 we also used the Partial Coefficient (the results are shown in Table 3 ). SPD correlated positively with all of the nine dimensions, but with weak coefficients, while ASPD correlated only with Somatization, Anxiety, Depression, Hostility, Phobic anxiety, Paranoid ideation, Psychoticism. Although these preliminary findings show that both scales are related to the means of assessing 
psychiatric problems and symptoms of psychopathology, the magnitude of the correlations is rather low (with none of them exceeding the level of .5 , required for a moderate relationship). Also, the correlations indices are similar for SPD and ASPD scales. We also conducted a correlation analysis between Global Severity Index, SPD and ASPD scores (Table 3). The results describe the same pattern of positive, significant, but low and similar coefficients.

Table 3. Partial Correlations between PDs scales and SCL-90 dimensions with DA-Desirability as control variable

\begin{tabular}{lll}
\hline & SPD & ASPD \\
\hline SCL-Somatization & $0,274^{*}$ & $0,234^{* *}$ \\
SCL-Obsessive-compulsive & 0,186 & 0,169 \\
SCL-Interpersonal sensitivity & $0,204^{*}$ & 0,127 \\
SCL -Depression & 0,195 & 0,18 \\
SCL-Anxiety & 0,2 & $0,286^{* *}$ \\
SCL-Hostility & $0,231^{*}$ & $0,23^{*}$ \\
SCL-Phobic anxiety & $0,248^{*}$ & $0,247^{*}$ \\
SCL-Paranoid ideation & $0,321^{* *}$ & $0,271^{* *}$ \\
SCL-Psychoticism & $0,241^{*}$ & $0,2 *$ \\
SCL -Global Index & $0,274^{*}$ & $0,261^{*}$ \\
\hline
\end{tabular}

Notes: $* p<.05 ; * * p<.01$.

Table 4. Factor Structure - DA ${ }^{12 \text { profile }}$, ASPD, SPD

\begin{tabular}{|c|c|c|c|c|}
\hline & \multicolumn{4}{|c|}{ Component } \\
\hline & 1 & 2 & 3 & 4 \\
\hline DA-Neuroticism & 0,864 & & & \\
\hline DA-Dysthymia & 0,795 & & & \\
\hline DA-Anxiety & 0,735 & & & \\
\hline DA-Dependency & 0,477 & & & \\
\hline Antisocial & & 0,692 & & \\
\hline DA-Demonstrativeness & & 0,633 & & \\
\hline DA-Hyperthymia & & 0,506 & 0,324 & 0,323 \\
\hline Schizoid & 0,452 & 0,501 & & \\
\hline DA-Hiper-perseverance & & 0,44 & & $-0,312$ \\
\hline DA-Emotivity & & & 0,844 & \\
\hline DA-Exaltation & & & 0,73 & \\
\hline DA-Lability & & & 0,546 & $-0,324$ \\
\hline DA-Uncontrollability & & & 0,458 & \\
\hline DA-Desirability & & & & 0,599 \\
\hline DA-Hyper exactness & & & & 0,397 \\
\hline
\end{tabular}

Notes: Extraction Method: Principal Axis Factoring; Rotation Method: Oblimin with Kaiser Normalization.

Exploratory factor analysis

To check how the two new scales (SPD and ASPD) are integrated into a general factorial structure of personality assessment, we computed a second order exploratory factor analysis using the 13 factors of $\mathrm{DA}^{12 \text { profile }}$ and the present scales. Using Principal Axis Factoring as the extraction method, we found that by introducing the two new scales into the analysis, the factorial model retained its four factors structure found by Constantin and his colleagues (2010) in a previous study regarding the factor structure of $\mathrm{DA}^{12 \text { profile }}$. The four factors explain a total of 68.31 of the variance.. Bartlett's Test of Sphericity was significant and the KMO Measure of Sampling Adequacy was .81. Consequently the model was accepted. Schizoid correlated with two factors, but it was better saturated in the second factor, where Antisocial also found its place (Table 4).

Two factors ("Negative Emotion", containing Neuroticism, Dysthymia, Dependency and Anxiety and "Impulsivity", containing Emotivity, Exaltation, Lability and Uncontrollability) retained their structure from the previous study (Constantin et al., 2010). Despite the fact that the Schizoid Scale could be included in the "Negative Emotion" factor, both of the two new scales were best saturated in a factor previously named "Behavioral Rigidity", also grouping Demonstrativeness, Hyperthymia and Hyper-perseverance. The last factor, named "Narcissistic Energy" group Desirability and Hyperexactness.

\section{Discussion}

Our first goal was to create a new and reliable way to measure schizoid and antisocial traits. The Reliability analysis shows that the SPD scale has an optimal level of internal consistency, while ASPD scale has a satisfactory one.

A second goal was to verify the correlations with other instruments that measure associated concepts in order to test the concurrent validity for our two scales. Both of them correlate with $\mathrm{DA}^{12 \text { profile }}$ Personality Inventory traits (Constantin et al., 2010), and Symptom Check List-90 as well (Derogatis, 1977).

Based on the analysis of the significant correlations (with average value) between ASPD scales and sub-scales of the SCL-90, we conclude that an antisocial individual is described as hostile and aggressive, alienated from his peers. Also, he might have traces of paranoid ideation about the police or beliefs that all of his problems are caused by other people. On the other hand, based on similar analyses, Schizoid individuals do not feel the need to socialize with other people, can become hostile towards them if they feel that their personal space has been invaded and might become anxious around other people.

Similarly, we expected for ASPD to correlate with hostility, paranoid ideation and psychoticism. Still, the magnitude of these correlations was rather low. Taking into considerations that SCL-90 scores reflect the momentary state of the individual and is not a personality inventory and that the correlations between the scales and the GSI are also significant, we can assume that our scale can generally evaluate psychological symptomatology, but further validation is necessary in order to use it for the assessment of specific issues and symptoms.

Unexpectedly, the scales correlate between themselves at a medium level and the correlation pattern with the SCL90 Subscales is very similar. Firstly, we excluded the possibility of a common method bias. The correlation pattern can be explained by the similarities between the Antisocial and Schizoid Personality Disorders. Both disorders present symptoms of emotional detachment, coldness and low empathy. We explored these characteristics with items such as "I feel better when I am alone", "People see me as cold and distant person" for the 
SPD scale and " I do not think too much about other people's feelings and opinions", "I do not understand why should I feel sorry for manipulating the week" for ASPD. Also, past research (Coid \& Ullrich, 2010) suggests that people with psychopathic symptoms (operationalised as a more extreme form of ASPD) have more chances of suffering from SPD than people without such symptoms. Also, SPD was associated with a number of antisocial behaviors, like kidnap, burglary and theft (Davison \& Janca, 2012). All these aspects could explain the medium correlation between the two scales and the correlation pattern with the SCL-90 subscales. The magnitude of the correlation between SPD and ASPD raises another common problem of the current classification system. The co-morbidity between various personality disorders in high and many researchers are willing to eliminate the current system and introduce a new one. In order to strengthen the clinical utility, the new ICD 11 will feature different levels of severity affecting four domains of psychological wellbeing (Tyrer, 2013).

Using the DA ${ }^{12 \text { profile }}$ Personality Inventory (Constantin et al., 2010) in order to assess the convergent and discriminant validity, ASPD had positive correlations with most of the dimensions. Taking into account only the medium correlations between the two sets of variables, we can create a profile of an antisocial individual. They behave theatrically, with a tendency towards lying and exaggerating things for personal gain (Demonstrativeness), are easily offendable and vengeful (Hyper-perseverance), impulsive, aggressive, centered on present needs (Uncontrollability), unpredictable in their behavior and emotions (Lability) and uncertain of their decisions (Dependency).

SPD correlated positively with most of the dimensions of $\mathrm{DA}^{12 \text { profile }}$. According to our data, a schizoid person can be hyper-perseverant, uncontrollable and labile person, and with lighter tendencies toward dysthymia and neurosis. As expected there are no correlations between SPD trait and Demonstrativeness, Exaltation, Emotivity and Desirability.

Although some of the correlations between our two scales and SCL-90 or DA ${ }^{12 \text { profile }}$ support the construct validity of the two new scales, others offer contradictory results. As such, the issue is not clear and needs further discussions. Moreover, we intend to reduce the number of items to 12 and, after another study, to compute the correlation pattern again.

The factor analysis shows that the Antisocial Scale can be grouped in factor also containing Demonstrativeness, Hyperthymia and Hyper-perseverance (called "Behavioral Rigidity"). An antisocial individual is always ready to lie and to exaggerate things into his advantage (as such, he shows traces of Demonstrativeness), can be vengeful and extract his "justice" after long periods of time (hyperperseverant) and when he needs it, he can be dynamic and energetic, especially in regards to superficial and present needs (hyperthymic).

The Schizoid Scale could have been grouped in two factors and the loading scores made it more suitable for "Behavioral Rigidity". The statistical result can be explained by the strong correlation between Schizoid and Antisocial Scales, even though the conceptual reason for this is less apparent.

Conclusions

When interpreting the results we need to take into account the limitations of our research. Firstly, we did not have a large sample. Moreover, all the participants were from a non-patient population, which may lead to a restriction of range. Given the prevalence of the personality disorders in the general population (10-14\%), our result could be generated by a small subsample of the participants. In order to further validate the scales, we need to assess the symptoms of SPD and ASPD in a patient population as well and to verify how well they can predict actual diagnosis of the two personality disorders. Secondly, the correlation matrix between the two scales, the factors of SCL-90 and those of DA ${ }^{12 p r o f i l e}$ has shown some unexpected result. After eliminating the possibilities of either a common method bias or a social desirability bias, we offered some explanations for our results. Still, we cannot fully support the construct validity of our scales.

According to our knowledge, this is the first Romanian attempt to create an assessment scale for personality disorders. This study is part of a longer endeavor, and we intend to develop reliable and valid instruments for each PD, starting from the way the DSM 5 and ICD-10 describe them. We relied our conceptualization of Antisocial Personality Disorder and Schizoid Personality Disorder on the information from the two manuals and, finally, we have evaluated two scales and a sum of 44 items. Both scales can be used by researchers, but we need to refine and retest them on a clinical sample in order to recommend them for psychiatric use.

\section{References}

American Psychiatric Association. (1952). Diagnostic and statistical manual of mental disorders. Washington, DC: Author.

American Psychiatric Association. (1980). Diagnostic and statistical manual of mental disorders (3rd ed.). Washington, DC: Author.

American Psychiatric Association. (2013). Diagnostic and statistical manual of mental disorders (5th ed.). Arlington, VA: American Psychiatric Publishing.

Bleuler, E. (1922). Die probleme der schizoidie und der syntonie. Zeitschrift fuer die gesamte. Nurologie und Psychiatrie, 78, 373-388.

Coid, J. \& Ullrich, S. (2010). Antisocial personality disorder is on a continuum with psychopathy. Comprehensive Psychiatry, 51(4), 426-433.

Constantin T, Hojbotă A, M., Niculescu A., Nechita V., Amariei C., Macarie A., (2010), Caracteristici psihometrice ale Chestionarului D.A. 307 - „Dimensiuni Accentuate", varianta 3.07(in English: Psychometric characteristics of the D.A. 307 Questionnaire - "Accented Dimensions" version 3.07), Psihologia Resurselor Umane, $8(1), 10-26$.

Costa Jr, P. T., \& McCrae, R. R. (1992). Neo Personality Inventory-Revised (NEO-PI-R) and Neo Five-Factor Inventory (NEO-FFI) professional manual. Odessa, FL: Psychological Assessment Resources.

Davison, S. \& Janca, A. (2012). Personality disorder and criminal behaviour. Current Opinion in Psychiatry, 25(1), $39-45$.

Derogatis, L. R. (1977). The SCL-90 Manual I: Scoring, administration and procedures for the SCL-90. Baltimore, MD: Clinical Psychometric Research.

Ekselius, L., Tillfors, M., Furmark, T., \& Fredrikson, M. (2001). Personality disorders in the general population: DSM-IV and ICD-10 defined prevalence as related to sociodemographic profile. Personality and Individual Differences, 30(2), 311-320. 
Grant, B., Hasin, D., Stinson, F., Dawson, D., Chou, S., Ruan, W., \& Pickering, R. (2004). Prevalence, Correlates, and Disability of Personality Disorders in the United States. The Journal of Clinical Psychiatry, 65(7), 948-958.

Hare, R. D. (1980). The assessment of psychopathy in criminal populations. Personality and Individual Differences, 1, 111-119.

Hare, R. D. (1985). Comparison of procedures for the assessment of psychopathy. Journal of Consulting and Clinical Psychology, 53, 7-16.

Harman, H. H. (1960). Modern factor analysis. Chicago, IL. University of Chicago Press.

Hoch, A. (1910). Constitutional factors in the dementia praecox group. Review of Neurology and Psychiatry, 8, 463-475.

Houser, Mallory C. (2015). A history of antisocial personality disorder in the diagnostic and statistical manual of mental illness and treatment from a rehabilitation perspective. Academic Dissertation, Southern Illinois University, Carbondale.

Holi, M. (2003). Assessment of psychiatric symptoms using the SCL-90. Academic Dissertation. University of Helsinki, Helsinki.

Leonhard, K., (1979). Personalități accentuate în viață și in literatură. Bucureşti: Editura Enciclopedică Română

Markon, K., Krueger, R., \& Watson, D. (2005). Delineating the Structure of Normal and Abnormal Personality: An Integrative Hierarchical Approach. Journal of Personality and Social Psychology, 88(1), 139-157.

Miller, M. B., Useda, J. D., Trull, T. J., Burr, R. M., \& MinksBrown, C. (2002). Paranoid, schizoid, and schizotypal personality disorders, in Adams, H. E., \& Sutker, P. B. (Eds.). Comprehensive handbook of psychopathology. Springer Science \& Business Media.
Ribot, T. (1890). Psychologie des sentiments. Paris: Delahaye and Lecrosnier.

Samuel, D. \& Widiger, T. (2008). A meta-analytic review of the relationships between the five-factor model and DSMIV-TR personality disorders: A facet level analysis. Clinical Psychology Review, 28(8), 1326-1342.

Schmieschek, H. (1970). Questionnaire for the determination of accentuated personalities. Psychiatrie, Neurologie, und medizinische Psychologie, 22(10), 378.

Sutker, P. B., \& Allain Jr, A. N. (2002). Antisocial Personality Disorder in Adams, H. E., \& Sutker, P. B. (Eds.). Comprehensive handbook of psychopathology. Springer Science \& Business Media.

Torgersen, S., Kringlen, E., \& Cramer, V. (2001). The Prevalence of Personality Disorders in a Community Sample. Archives of General Psychiatry, 58(6), 590.

Tyrer, P. (2013). The classification of personality disorders in ICD-11: Implications for forensic psychiatry. Criminal Behaviour and Mental Health, 23(1), 1-5.

Widiger, T. \& Costa, P. (1994). Personality and personality disorders. Journal of Abnormal Psychology, 103(1), 7891.

Widiger, T. A., Trull, T. J., Clarkin, J. F., Sanderson, C., \& Costa, P. T., Jr. (1994). A description of the DSM-III-R and DSM-IV personality disorders with the five factor model of personality. In P. T. Costa, Jr. \& T. A. Widiger (Eds.), Personality disorders and the five factor model of personality. Washington, DC: American Psychological Association.

Widiger, T., Trull, T. (2007). Plate tectonics in the classification of personality disorder: Shifting to a dimensional model. American Psychologist, 62(2), 71-83.

World Health Organization. International Classification of Diseases, 10th Revision. Mental and Behavioural Disorders. Geneva: World Health Organization, 1992. 